\title{
COVID-19 Vaccine Uptake Among People Living with HIV
}

\author{
Tim W. Menza ${ }^{1,2}$ (D) Jeff Capizzi ${ }^{1} \cdot$ Amy I. Zlot ${ }^{1} \cdot$ Michelle Barber $^{1} \cdot$ Lea Bush $^{1}$ \\ Accepted: 24 December 2021 / Published online: 7 January 2022 \\ (c) The Author(s), under exclusive licence to Springer Science+Business Media, LLC, part of Springer Nature 2021
}

\begin{abstract}
People living with HIV (PLWH) are at greater risk for severe COVID-19 and are a priority population for COVID-19 vaccination. As of June 15, 2021, 61.6\% of PLWH in Oregon received $\geq 1$ COVID-19 vaccine dose. Younger PLWH, Hispanic/ Latinx PLWH and PLWH who inject drugs or reside in rural and frontier areas had low vaccine uptake while PLWH who were engaged in care, enrolled in the AIDS Drug Assistance Program, and vaccinated against influenza had high vaccine uptake. Greater advocacy, education, and care navigation are required to increase COVID-19 vaccine access and uptake among PLWH.
\end{abstract}

Keywords HIV $\cdot$ COVID-19 $\cdot$ Vaccination $\cdot$ Public health surveillance

\section{Introduction}

Mounting evidence indicates that people living with HIV (PLWH) are at higher risk of severe COVID-19 [1]. First, PLWH who are not on antiretroviral therapy, have detectable viral loads, and/or have lower CD4 counts are more likely to experience a dysregulated immune response to SARS-CoV-2 infection [1,2]. Second, more than 50\% of PLWH in Oregon and the United States are over age 50 [3]. Third, PLWH are more likely to experience comorbid medical conditions and experience them at a younger age compared to those without HIV [4]. Fourth, PLWH are more likely to experience social and economic disadvantage than those without HIV [5] and this disadvantage is associated with a greater risk of COVID-19 [6]. Finally, HIV is more common among Black, Hispanic/Latinx, Indigenous and LGBTQ + communities all of whom have shouldered the burden of the COVID-19 pandemic in Oregon and across the United States [7, 8]. Thus, PLWH are made vulnerable to COVID-19 through complex, intersecting biological and structural mechanisms. The increased risk of severe COVID-19 makes access to and

Tim W. Menza

timothy.w.menza@state.or.us

1 Public Health Division, Oregon Health Authority, 800 NE Oregon Street, Portland, OR 97232, USA

2 Division of Infectious Disease, Department of Medicine, Oregon Health and Science University, Portland, OR 97239, USA uptake of COVID-19 vaccination a priority for PLWH. We assessed COVID-19 vaccine uptake and correlates of vaccination among PLWH in Oregon.

\section{Methods}

\section{Data Sources and Analytic Sample}

HIV surveillance data. As of May 10, 2021, 8,122 living Oregon residents with laboratory-confirmed HIV were reported to the Oregon Health Authority's (OHA) HIV Surveillance Program. For this analysis, we excluded cases $<18$ years old $(n=31)$ and cases diagnosed in $2021(n=69)$. The final sample represented 8022 Oregon PLWH $\geq 18$ years old diagnosed as of December 31, 2020 and alive as of May 10, 2021.

Immunization data. In Oregon, COVID-19 vaccination was first available to healthcare workers on December 12, 2020; to people 65 years of age and older on January 25 , 2021; and to people 45-64 years of age and 16-44 years of age with underlying medical conditions on March 29, 2021 and April 5, 2021, respectively. The date of administration and number of doses of mRNA (Pfizer/BioNTech and Moderna) and adenovirus-26 vector (Johnson \& Johnson) vaccines were ascertained through the ALERT immunization information system (IIS). All COVID-19 vaccine providers were required to submit vaccination information to ALERT IIS. 
We matched HIV surveillance records to ALERT IIS through a deterministic match using name, date of birth, address, and phone number. Matching was performed on June 15,2021 . Ninety percent of HIV cases had a record in ALERT IIS.

\section{Outcome and Covariates}

The outcome of interest was the percentage of PLWH who received at least one COVID-19 vaccine dose. We examined the following covariates: sex at birth (male, female); age in years $(18-29 ; 30-39 ; 40-49 ; 50-59 ; 60-69 ; 70$ and older); race/ethnicity (American Indian/Alaska Native; Asian; Black; Hispanic/Latinx; Native Hawaiian/Pacific Islander; white; multiracial); CDC transmission risk (men who have sex with men [MSM]; MSM who use injection drugs [MSM/IDU]; injection drug use [IDU]; heterosexual; unknown risk; and other risk); urbanicity of zip code of residence (urban, rural, frontier); year of diagnosis (prior to $1997 ; 1997$ to $2010 ; 2011$ to present); HIV viral load or CD4 count measured in 2020 (a proxy for care engagement; no, yes); enrollment in Oregon State AIDS Drug Assistance Program (ADAP; no, yes); and, receipt of the influenza vaccine in 2019-2020 (no, yes). We also examined the most recent HIV viral load ( $<200$ copies $/ \mathrm{mL}, \geq 200$ copies $/ \mathrm{mL}$ ) and CD4 count $\left(\geq 200\right.$ cells $/ \mathrm{mm}^{3},<200$ cells $/ \mathrm{mm}^{3}$ ) among those with non-missing values in 2020 .

\section{Statistical Analysis}

We calculated the percentage of PLWH with at least one COVID-19 vaccine dose overall and by each covariate. We used negative binomial regression with a log link and robust standard errors to estimate prevalence ratios (PRs) and 95\% confidence intervals (CIs) in bivariable models. Covariates with $P<0.25$ in bivariable models were included in a multivariable model. Statistical significance for the multivariable model was defined at the $P<0.05$ level. We examined the values of HIV viral load and CD4 count in bivariable models only. We used SPSS v28 (IBM Corporation, Airmonk, NY) for all analyses.

\section{Results}

Of 8,022 PLWH, 4943 (61.6\%; 95\%CI 60.5-62.7\%) received at least one COVID-19 vaccine dose and 4538 (56.6\%; 95\%CI 55.5-57.5\%) received a complete vaccine series, as of June 15, 2021 (Table 1, Supplemental Figure). In comparison, 2,228,497 (65.6\%; 95\%CI 65.5\%, 65.7\%) and $2,141,591(63.1 \%$; 95\%CI $63.0 \%, 63.1 \%)$ of $3,394,960$ Oregonians without HIV received at least one COVID-19 vaccine dose and a complete series, respectively $(P<0.001$ for pairwise comparisons between PLWH and Oregonians without HIV with at least one dose and a complete series, respectively).

The percentage of PLWH who received at least one COVID-19 vaccine dose ranged from $30.9 \%$ among those without a CD4 count and viral load measurement in 2020 to $78.1 \%$ among those who received an influenza vaccine in 2019-2020. Compared to PLWH 18-29 years old, vaccination was more likely among PLWH aged 50 years old and older. Hispanic/Latinx PLWH were less likely to be vaccinated than white PLWH while MSM were more likely to be vaccinated compared to PLWH who use injection drugs (PWID). Vaccination was less likely among PLWH residing in rural zip codes compared to PLWH residing in urban zip codes. A greater percentage of PLWH with a CD4 count or viral load measurement in 2020 were vaccinated than PLWH without these labs. PLWH enrolled in ADAP and who received an influenza vaccine in 2019-2020 were more likely to be vaccinated than those not enrolled in ADAP and who did not receive an influenza vaccine, respectively.

Among the 6137 PLWH with a CD4 count in 2020, vaccination was more likely among those with CD4 counts $\geq 200$ cells $/ \mathrm{mm}^{3}$ (3939/5789; 68.0\%) compared to those with CD4 counts $<200$ cells $/ \mathrm{mm}^{3}$ (203/348; 58.3\%, $\left.P=0.032\right)$. Among the 6441 PLWH with a viral load in 2020, vaccination was more likely among those with viral loads $<200$ copies $/ \mathrm{mL}$ (4286/6057; 70.8\%) compared to those with viral loads $\geq 200$ copies $/ \mathrm{mL}(183 / 384 ; 47.7 \%, P<0.001)$.

\section{Discussion}

Just over three-fifths of PLWH in Oregon received at least one dose of a COVID-19 vaccine as of June 15, 2021. While COVID-19 hospitalizations are increasing among younger adults [9], COVID-19 vaccination uptake was lowest among PLWH 18-29 years old. Early vaccine eligibility prioritized older adults but a quarter of younger adults reported that they would probably or definitely not get vaccinated citing concerns about safety and side effects [10]. PLWH of color overall, and Hispanic/Latinx PLWH in particular, were less likely to be vaccinated compared to white PLWH. Building trust in COVID-19 vaccination among PLWH of color requires not only coordinated, broad-based grassroots vaccine advocacy, but also authentic community investment that acknowledges experiences of racism in health care [11]. Vaccine uptake among PWID was low. Harm reduction programs have proven essential during the COVID-19 pandemic and the integration of vaccination into syringe service programs may be key to enhancing vaccine access and uptake among PWID. In addition, PLWH in rural and frontier areas, where vaccine availability is sparser and vaccine hesitancy is higher [12], were less likely to be vaccinated compared to PLWH in urban areas. 
Table 1 Bivariable and multivariable models of COVID-19 vaccination prevalence among people living with HIV as of June 15, 2021, Oregon

\begin{tabular}{|c|c|c|c|c|c|c|}
\hline & $N(\%)$ population & $N(\%) \geq 1$ dose & Crude PR (95\%CI) & $P$ value & Adjusted PR (95\%CI) & $P$ value \\
\hline Overall & $8022(100)$ & $4943(61.6)$ & & & & \\
\hline \multicolumn{7}{|l|}{ Socio-demographics } \\
\hline \multicolumn{7}{|l|}{ Sex at birth } \\
\hline Male & $7095(88.4)$ & $4460(62.9)$ & REF & & REF & \\
\hline Female & $927(11.6)$ & $483(52.1)$ & $0.83(0.75,0.91)$ & $<0.001$ & $0.96(0.81,1.15)$ & 0.962 \\
\hline \multicolumn{7}{|l|}{ Age } \\
\hline $18-29$ & $476(5.9)$ & $216(45.4)$ & REF & & REF & \\
\hline $30-39$ & 1409 (17.6) & $702(49.8)$ & $1.10(0.94,1.28)$ & 0.230 & $1.03(0.85,1.25)$ & 0.766 \\
\hline $40-49$ & $1682(21.0)$ & $972(57.8)$ & $1.27(1.10,1.47)$ & 0.001 & $1.17(0.96,1.42)$ & 0.117 \\
\hline $50-59$ & $2531(31.6)$ & $1671(66.0)$ & $1.45(1.26,1.67)$ & $<0.001$ & $1.24(1.02,1.50)$ & 0.033 \\
\hline $60-69$ & $1443(18.0)$ & $1026(71.1)$ & $1.57(1.35,1.81)$ & $<0.001$ & $1.29(1.05,1.58)$ & 0.017 \\
\hline 70 and elder & $481(6.0)$ & $356(74.0)$ & $1.63(1.38,1.93)$ & $<0.001$ & $1.27(1.00,1.61)$ & 0.048 \\
\hline \multicolumn{7}{|l|}{ Race/ethnicity } \\
\hline American Indian/Alaska Native & $76(0.9)$ & $38(50.0)$ & $0.76(0.55,1.05)$ & 0.099 & $0.84(0.56,1.25)$ & 0.394 \\
\hline Asian & $176(2.2)$ & $112(63.6)$ & $0.97(0.81,1.17)$ & 0.773 & $1.00(0.76,1.30)$ & 0.979 \\
\hline Black/African American & $631(7.9)$ & $330(52.3)$ & $0.80(0.71,0.89)$ & $<0.001$ & $0.91(0.78,1.06)$ & 0.221 \\
\hline Hispanic/Latinx & $1181(14.7)$ & $591(50.0)$ & $0.76(0.70,0.83)$ & $<0.001$ & $0.81(0.71,0.92)$ & 0.002 \\
\hline Native Hawaiian/Pacific Islander & $24(0.3)$ & $13(54.2)$ & $0.83(0.48,1.43)$ & 0.497 & $1.00(0.50,2.01)$ & 0.996 \\
\hline White & $5781(72.1)$ & $3782(65.4)$ & REF & & REF & \\
\hline Multiracial & $153(1.9)$ & $71(50.3)$ & $0.77(0.61,0.96)$ & 0.023 & $0.91(0.69,1.21)$ & 0.912 \\
\hline \multicolumn{7}{|l|}{ CDC HIV transmission risk } \\
\hline MSM & $5254(65.5)$ & $3522(67.0)$ & REF & & REF & \\
\hline MSM/IDU & $751(9.4)$ & $414(55.1)$ & $0.82(0.74,0.91)$ & $<0.001$ & $0.85(0.75,0.97)$ & 0.015 \\
\hline IDU & $643(8.0)$ & $269(41.8)$ & $0.62(0.55,0.71)$ & $<0.001$ & $0.68(0.57,0.80)$ & $<0.001$ \\
\hline Heterosexual & $767(9.6)$ & $430(56.1)$ & $0.84(0.76,0.92)$ & $<0.001$ & $0.90(0.74,1.08)$ & 0.255 \\
\hline Unknown & $542(6.8)$ & $282(52.0)$ & $0.78(0.69,0.88)$ & $<0.001$ & $0.88(0.75,1.04)$ & 0.129 \\
\hline Other & $65(0.8)$ & $26(40.0)$ & $0.60(0.41,0.88)$ & 0.009 & $0.70(0.43,1.13)$ & 0.141 \\
\hline \multicolumn{7}{|l|}{ Zip code urbanicity } \\
\hline Urban & $6445(80.3)$ & $4123(64.0)$ & REF & & REF & \\
\hline Rural & $1485(18.5)$ & $780(52.5)$ & $0.82(0.76,0.89)$ & $<0.001$ & $0.87(0.79,0.96)$ & 0.008 \\
\hline Frontier & $92(1.1)$ & $40(43.5)$ & $0.68(0.50,0.92)$ & 0.015 & $0.81(0.55,1.19)$ & 0.289 \\
\hline \multicolumn{7}{|l|}{ Country of birth } \\
\hline United States & $7073(88.2)$ & $4422(62.5)$ & REF & & REF & \\
\hline Outside the United States & $949(11.8)$ & $521(54.9)$ & $0.88(0.80,0.96)$ & 0.005 & $1.02(0.88,1.19)$ & 0.740 \\
\hline \multicolumn{7}{|l|}{ Clinical characteristics } \\
\hline \multicolumn{7}{|l|}{ Year of diagnosis } \\
\hline Before 1997 & $1765(22.0)$ & $1238(70.1)$ & $1.29(1.19,1.39)$ & $<0.001$ & $1.01(0.92,1.12)$ & 0.785 \\
\hline 1997-2010 & $3694(46.0)$ & $2308(62.5)$ & $1.15(1.07,1.22)$ & $<0.001$ & $1.03(0.91,1.16)$ & 0.685 \\
\hline After 2010 & $2563(31.9)$ & $1397(54.5)$ & REF & & REF & \\
\hline \multicolumn{7}{|c|}{ CD4 count or viral load measurement, 2020} \\
\hline No & $1313(16.4)$ & $406(30.9)$ & REF & & REF & \\
\hline Yes & $6709(83.6)$ & 4537 (67.6) & $2.19(1.98,2.42)$ & $<0.001$ & $1.62(1.43,1.84)$ & $<0.001$ \\
\hline \multicolumn{7}{|l|}{ ADAP enrollment, 2020} \\
\hline No & $4125(51.4)$ & $2282(55.3)$ & REF & & REF & \\
\hline Yes & $3897(48.6)$ & $2661(68.3)$ & $1.23(1.17,1.30)$ & $<0.001$ & $1.08(1.01,1.17)$ & 0.033 \\
\hline \multicolumn{7}{|l|}{ Flu vaccination, 2019-2020 } \\
\hline No & $2957(36.9)$ & $987(33.4)$ & REF & & REF & \\
\hline Yes & $5065(63.1)$ & $3956(78.1)$ & $2.34(2.18,2.51)$ & $<0.001$ & $2.03(1.86,2.22)$ & $<0.001$ \\
\hline
\end{tabular}

$A D A P$ AIDS Drug Assistance Program, $C I$ confidence interval, $I D U$ injection drug use, $M S M$ men who have sex with men, $P R$ prevalence ratio, $R E F$ referent 
PLWH who did not have a CD4 count or viral load in 2020, a standard surveillance proxy for care engagement, were less likely to be vaccinated than those who did. Many clinics deferred routine care for patients with well-controlled HIV and/or converted to telemedicine. For some PLWH, the risk of COVID-19 made attending clinic unsafe. Expansion of home- and communitybased vaccination programs may improve vaccine uptake among PLWH whose access to in-person care has been curtailed by COVID-19. Moreover, vaccine uptake was lower among those at greater risk of severe COVID-19 as defined by viral load and CD4 counts [1]. In addition, PLWH with lower CD4 counts may mount an inadequate response to vaccination and may require increased support to complete a newly recommended three-dose vaccination series for people with untreated or advanced HIV. Social determinants of health affecting uptake of COVID19 vaccination likely overlap with those affecting clinic attendance, antiretroviral adherence, and viral suppression [5]. In contrast, ADAP enrollment was associated with greater vaccine uptake; case workers facilitate access to vaccination through assistance with finding vaccination sites, appointment scheduling, and transportation. Finally, receipt of influenza vaccine was the strongest predictor of COVID-19 vaccine uptake. PLWH vaccinated against influenza may be more likely to adopt preventive health interventions and have more confidence in the effectiveness and safety of vaccines. While receipt of influenza vaccine may also indicate a high level of care engagement, the association between influenza vaccination and COVID-19 vaccination remained strong in a multivariable model that included a standard surveillance measure of care engagement.

There are limitations to this work. First, ALERT IIS only captures vaccines administered in Oregon; therefore, PLWH vaccinated out of state were not represented in these data. Second, reporting to ALERT IIS may not be $100 \%$ complete resulting in missing vaccination status for some PLWH. Third, because HIV surveillance does not collect clinical data on comorbidities, it was not possible to examine vaccine uptake by comorbidities that may increase COVID19 risk. Finally, measurements of CD4 count or viral load reported to the surveillance system are a proxy for care engagement and subject to misclassification. For example, PLWH may have had HIV-related labs drawn by a provider other than their HIV provider (e.g., an emergency room or urgent care provider). Thus, the report of a viral load or CD4 count in this setting may misclassify a patient as engaged in care. In addition, the presence of these measurements may represent either an in-person visit, a telemedicine visit with an associated laboratory visit, or just a laboratory visit. Each type of care engagement may have differential impact on vaccine uptake that is not captured in these data.
COVID-19 vaccination is critical to the survival of PLWH through this novel global pandemic. Younger PLWH, Hispanic/Latinx PLWH, PWID, who live in rural and frontier areas, who have not engaged in care or enrolled in ADAP, and who have not received an influenza vaccine may benefit from more intensive advocacy, outreach, and care navigation to increase COVID-19 vaccine uptake.

Supplementary Information The online version contains supplementary material available at https://doi.org/10.1007/s10461-021-03570-9.

Author Contributions TWM conceived the study, conceptualized the analyses, and wrote the manuscript; JC performed the analyses; AIZ provided critical feedback on the manuscript; MB performed the match between HIV and immunization surveillance data; LB provided critical feedback on the manuscript.

Funding No funding was received to assist with the preparation of this manuscript.

Data Availability Data is available upon reasonable request.

Code Availability No custom software application or code was used in the conduct of this study.

\section{Declarations}

Conflict of interest The authors have no relevant financial or non-financial interests to disclose.

Ethical Approval This manuscript reports data collected for public health surveillance and is not considered research. No individual's data or image is presented in this manuscript.

\section{References}

1. Sun J, Patel RC, Zheng Q, Madhira V, Olex AL, Islam JY, et al. COVID-19 disease severity among people with HIV infection or solid organ transplant in the United States: a nationally-representative, multicenter, observational cohort study. 2021. https://doi. org/10.1101/2021.07.26.21261028v1

2. Meckiff BJ, Ramírez-Suástegui C, Fajardo V, Chee SJ, Kusnadi A, Simon H, et al. Imbalance of regulatory and cytotoxic SARSCoV-2-reactive CD4+ T cells in COVID-19. Cell. 2020;183:13401353.e16.

3. Centers for Disease Control and Prevention. HIV Surveillance Report. 2019;2021(32):123.

4. Nanditha NGA, Paiero A, Tafessu HM, St-Jean M, McLinden T, Justice AC, et al. Excess burden of age-associated comorbidities among people living with HIV in British Columbia, Canada: a population-based cohort study. BMJ Open. 2021;11:e041734.

5. Menza TW, Hixson LK, Lipira L, Drach L. Social determinants of health and care outcomes among people with HIV in the United States. Open Forum Infect Dis. 2021. https://doi.org/10.1093/ofid/ ofab330.

6. De Ridder D, Sandoval J, Vuilleumier N, Azman AS, Stringhini S, Kaiser L, et al. Socioeconomically disadvantaged neighborhoods face increased persistence of SARS-CoV-2 clusters. Front Public Health. Frontiers 2021. https://doi.org/10.3389/fpubh.2020. 626090/full 
7. Krause KD. Implications of the COVID-19 pandemic on LGBTQ communities. J Public Health Manag Pract. 2021;27:S69.

8. Romano SD. Trends in racial and ethnic disparities in COVID19 hospitalizations, by region-United States, March-December 2020. MMWR Morb Mortal Wkly Rep. 2021;70.

9. COVID-19 hospitalizations. https://gis.cdc.gov/grasp/covidnet/ COVID19_5.html. Accessed 20 Aug 2021

10. Baack BN. COVID-19 vaccination coverage and intent among adults aged 18-39 years-United States, March-May 2021. MMWR Morb Mortal Wkly Rep. 2021;70. https://www.cdc.gov/ mmwr/volumes/70/wr/mm7025e2.htm. Accessed 20 Aug 2021

11. Ojikutu BO, Stephenson KE, Mayer KH, Emmons KM. Building trust in COVID-19 vaccines and beyond through authentic community investment. Am J Public Health. 2021;111:366-8.
12. KFF COVID-19 Vaccine Monitor- Rural America. KFF. 2021. https://www.kff.org/coronavirus-covid-19/poll-finding/kff-covid19-vaccine-monitor-rural-america/. Accessed 20 Aug 2021

Publisher's Note Springer Nature remains neutral with regard to jurisdictional claims in published maps and institutional affiliations. 